\title{
POLÍTICA PÚBLICA EDUCATIVA CENTRADA EN LA TECNOLOGÍA. EL CASO DEL PROGRAMA HABILIDADES DIGITALES PARA TODOS EN EL ESTADO DE PUEBLA
}

\section{Claudia Rivera Hernández}

Investigadora del Instituto de Ciencias de Gobierno y Desarrollo Estratégico de la Benemérita Universidad Autónoma de Puebla. Postdoctora en Educación Superior y Política Pública, Universidad de Nuevo México, USA. claudia.rivera@correo.buap.mx

\section{Valentina Campos Cabral}

Profesora de la Facultad de Derecho y Ciencias Sociales de la Benemérita Universidad Autónoma de Puebla. Posdoctora por la UNAM y la BUAP. Doctora en Estrategias para el Desarrollo por el Colegio de Postgraduados. valiacc@gmail.com

\section{Resumen}

El objetivo general de este documento es analizar la implementación de la política pública educativa para el uso de la tecnología como instrumento de enseńanza. En particular se detalla la implementación del Programa en Habilidades Digitales para Todos, en el Estado de Puebla con el fin de identificar el avance o retroceso de la brecha digital a través de una política pública; también busca de manera secundaria identificar los procesos de formación de cohesión social entre los usuarios del componente de capacitación. Los hallazgos encontrados arrojan que, a pesar de la limitada reducción de la brecha de conocimientos producto de la capacitación en $\mathrm{Ha}$ bilidades Digitales para Todos, si se generaron estrategias institucionales que favorecieron la inclusión y que fueron producto de la autogestión de los participantes en dicho programa, condiciones claves para la existencia de cohesión social.

Palabras clave: brecha digital, cohesión social, política pública educativa, TIC. 
Política pública educativa centrada en la tecnología. El caso del programa habilidades digitales para todos en el estado de puebla y su efecto en la formación de cohesión social.

\section{EDUCATIONAL PUBLIC POLICY CENTERED IN TECHNOLOGY. THE CASE OF THE PROGRAM DIGITAL SKILLS FOR ALL IN THE STATE OF PUEBLA}

\section{Abstract}

The general objective of this document is to analyze the implementation of public education policy for the use of technology as a teaching tool. In particular, the implementation of the Digital Skills for All Program in the State of Puebla is detailed in order to identify the advance or retreat of the digital divide through a public policy; it also seeks in a secondary way to identify the processes of social cohesion formation among the users of the training component. The findings show that despite the limited reduction of the knowledge gap resulting from the training in Digital Skills for All, if institutional strategies were generated that favored inclusion and were the product of the self-management of participants in said program, conditions keys for the existence of social cohesion.

Keywords: digital gap, social cohesion, public education policy, ITC.

\section{Introducción}

El desarrollo tecnológico y científico de la sociedad actual es cambiante y progresivo, exigiéndole al sistema educativo modificaciones importantes en el quehacer académico; estos cambios que requiere la educación en todos los niveles, se originan de los retos que enfrentan una economía globalizada y la competencia internacional de mercados. Por su parte, las brechas sociales generadas en los últimos años por el avance tecnológico, impiden que gran parte de la sociedad mexicana resuelva sus necesidades de acceso a la información: las nuevas comunicaciones han modificado la forma en que entendemos al mundo, y gracias a los avances tecnológicos, contamos con mayor capacidad de procesamiento de información. Dado que en nuestros días a la gente que se encuentra marginada del acceso a la tecnología se le considera tecnológicamente analfabeta, las políticas educacionales tendrán que promover el uso de la información y las comunicaciones a fin de resolver la inequidad que puede producir la falta de acceso a la tecnología. 
El uso de la tecnología ha impactado la vida cotidiana; la conectividad y el acceso a equipo de cómputo, son condiciones que no se han resuelto en México, donde a pesar de los múltiples esfuerzos que se realizan por parte del gobierno, no se ha logrado revertir las cifras de analfabetismo digital. Los pobres son cada vez menos capaces de incorporarse a la vida productiva de una manera adecuada pues sus carencias de información repercuten directamente en las condiciones de pobreza. Las causas más probables del ensanchamiento de la brecha digital son los bajos niveles de accesibilidad y conectividad, así como la falta de equipo, todo ello en función de los altos costos que representan.

A pesar de que el gobierno mexicano ha destinado una parte importante a la implantación de políticas tecnológicas, los niveles de pobreza en el país han aumentado, imposibilitando el cierre de la brecha. Las cifras publicadas por la última Encuesta Nacional sobre Disponibilidad y Uso de Tecnologías de la Información en los Hogares (EDUTIH), publicada por el INEGI (2016), mostró que los usuarios de computadoras se redujeron en 4.3 puntos porcentuales, mientras que los usuarios de internet y los usuarios de telefonía móvil celular, aumentaron dos puntos porcentuales respecto a la medición realizada en el año 2015 en cada una de dichas categorías. Esta cifra representa una reducción muy limitada en cuanto al acceso a la tecnología en México. Con relación al uso de internet, los datos publicados por la EDUTIH (INEGI, 2016), mostraron que del 2015 al 2016, el incremento más relevante se produjo en el uso para entretenimiento con un aumento de 8.7 puntos porcentuales, la actividad de ordenar o comprar productos aumentó 6.2 puntos porcentuales y el acceso a contenidos audiovisuales aumentó 5.3 puntos porcentuales. Estas tres categorías son las que tuvieron mayor avance en el uso de internet respecto al ańo 2015, y todas ellas están relacionadas con el ocio, teniendo un crecimiento total de $20.2 \%$. Por el contrario, las actividades que tuvieron el mayor retroceso en el uso de internet, son las relacionadas con la educación. Las cifras son relevantes pues la reducción del uso para apoyar la educación o la capacitación fue de 4.8 puntos porcentuales y la reducción en el uso de internet para la obtención de información, se redujo en 4.3 puntos porcentuales.

Lo anterior implica, que el acceso a la tecnología por parte de los mexicanos está relacionado con el entretenimiento y actividades de 
Política pública educativa centrada en la tecnología. El caso del programa habilidades digitales para todos en el estado de puebla y su efecto en la formación de cohesión social.

esparcimiento, mientras que los pobladores de otros países, utilizan la tecnología para mejorar su nivel educativo y su cultura. Por su parte, la política educativa mexicana en materia de acceso a la tecnología, ha generado diversos programas gubernamentales para reducir la brecha tecnológica con el fin de evitar que las carencias de información se amplíen. Por ello, este informe de investigación analiza el programa denominado "Habilidades Digitales para Todos (HDT)", que estuvo integrado por cuatro componentes: conectividad, infraestructura, capacitación y acompańamiento pues se considera que ha sido una de las estrategias más relevantes puesta en marcha por el gobierno federal para la reducción de las brechas digitales.

En particular, este trabajo tiene como objetivo general describir dicho programa y su aplicación en el Estado de Puebla, con el fin de identificar el avance o retroceso de la brecha digital a través de una política pública; también busca de manera secundaria identificar los procesos de formación de cohesión social entre los usuarios del componente de capacitación. La investigación está integrada por cinco secciones. En la sección II se presentan algunas consideraciones teóricas que permiten identificar la relación existente entre la cohesión social y la reducción de las brechas tecnológicas. En la sección III se presenta la operacionalización del programa HDT en el Estado de Puebla. En la sección IV se muestran los principales hallazgos, mismos que demuestran la existencia de cohesión social a pesar de existe poco avance en la reducción de las brechas digitales entre los profesores del magisterio poblano. En la quinta y última sección se presentan algunas reflexiones relevantes al trabajo.

\section{Consideraciones teóricas en la relación de la Cohesión social y la formación de competencias digitales}

Actualmente, millones de mexicanos se encuentran en pobreza alimentaria, de bienes y de conocimientos; El CONEVAL (Consejo Nacional de Evaluación de la Política de Desarrollo Social), considera a la pobreza como un problema multidimensional, resultado de la privación de las condiciones mínimas para que las personas puedan integrarse de manera productiva a la sociedad, de tal forma que un bajo nivel de ingreso acumulado en los hogares y nulo ejercicio de los derechos fundamentales, producen carencias socia- 
les (CONEVAL, 2012). Dos indicadores que evidencian estas carencias son: el rezago educativo promedio en el hogar y el grado de cohesión social. Por su parte, El Informe Anual sobre el Progreso y los Desafíos regionales de la Agenda 2030 para el Desarrollo Sostenible en América Latina y el Caribe publicado por la CEPAL en abril de 2017, destaca la poca capacidad de la región para competir de manera internacional, pues existe un notorio retraso en modernización tecnológica; se han reducido los presupuestos de inversión en investigación y desarrollo y las condiciones sociales se han pauperizado. En este sentido, el estudio de la cohesión social se vuelve prioritario con el fin de contrarrestar los efectos del resquebrajamiento social que la polarización económica produce.

Las Ciencias Sociales han sido el caldo de cultivo para innumerables estudios sobre cohesión social (Botterman, Hooghe y Reeskens, 2012; Demireva y McNeil, 2012; Jenson, 2010; Juul, 2010; Vasta, 2010). También existen investigaciones en los campos de la psicología (Cramm, Van Dijk, y Nieboer, 2013; Carrón, 1995), la medicina (Wilkinson, 1997; Kawachi y Kennedy 1997), incluso la zoología (Wartburton y Lazarus,1991; Wey y Limstein, 2010), entre otros autores, sin embargo, no existe un consenso mundial sobre el concepto de cohesión social, las definiciones abundan pues ha sido estudiado desde diversas perspectivas. Los estudios europeos se han centrado en los efectos de la cohesión social sobre las democracias y colectivos sociales; en los países anglosajones se han concentrado en evaluar los efectos individuales de la cohesión social; y la creciente corriente latinoamericana, ha centrado sus esfuerzos en identificar el papel gubernamental en el desarrollo de la cohesión social. (Rivera, 2017a). Así, se puede afirmar que en América Latina, los estudios sobre cohesión social se encuentran en creciente desarrollo y en los últimos años se han generado grandes avances para clarificar las representaciones sociales sobre este importante concepto. Rivera (2017b), explica que:

"En América Latina destacan los estudios publicados por la CEPAL. En 2007, el libro coordinado por Ernesto Ottone y Ana Sojo establece que la cohesión social es un elemento que permite fortalecer el tramado social mediante el sentido de pertenencia. Más tarde, en el año 2010, Juan Carlos Feres y Pablo Villatoro, definen un marco común de referencia para medir la cohesión 
Política pública educativa centrada en la tecnología. El caso del programa habilidades digitales para todos en el estado de puebla y su efecto en la formación de cohesión social. social... [la cual]...implica pensar en la manera que tienen los países para hacer frente a la pobreza y la desigualdad, sin que se vean afectados los lazos sociales y la democracia”. (Rivera, 2017b: 6-8).

Por su parte el programa para la cohesión social en América Latina "EuroSocial", (UE, 2013, s/p), establece en su portal web que la cohesión social es el atributo de las sociedades que implica la igualdad de oportunidades para que la población pueda ejercer sus derechos fundamentales y asegurar su bienestar, sin discriminación de ningún tipo y atendiendo a la diversidad. Desde una perspectiva individual, la cohesión social supone la existencia de personas que se sienten parte de una comunidad, participan activamente en diversos ámbitos de decisión y son capaces de ejercer una ciudadanía activa. La cohesión social también implica el desarrollo de políticas públicas y mecanismos de solidaridad entre individuos, colectivos, territorios y generaciones. Por su parte, la Comisión Económica para América Latina y el Caribe (CEPAL, 2007), incorporó la identificación de procesos y resultados específicos de cohesión, agrupándolos en tres componentes que se mencionan a continuación:

- Las distancias o brechas: que son resultados o expresiones visibles de la operación de los mecanismos de exclusión/inclusión y se refiere a las condiciones materiales en que viven los grupos y comunidades excluidos de la participación en actividades sociales esenciales para la vida, del ejercicio de sus derechos básicos y del acceso a los recursos y oportunidades necesarios para el desarrollo de sus potencialidades. Estas situaciones se manifiestan como brechas objetivas de bienestar. En comparación con las condiciones de vida de otros grupos sociales o considerando umbrales normativos de acceso a recursos o de garantía de derechos. Entre sus dimensiones se encuentran el empleo, los ingresos y la pobreza, la protección social, la educación, el acceso a las nuevas tecnologías, la salud, el consumo y la disponibilidad de servicios básicos.

- Los mecanismos institucionales de inclusión/exclusión: corresponden a las acciones ejecutadas por los distintos actores institucionales y que pueden repercutir en la estructura de oportunidades, en la acumulación de ventajas y desventajas y en los procesos y resultados de inclusión exclusión. En este ámbito, se otorga prioridad a las iniciativas explícitamente orientadas a promover la in- 
clusión y cohesión sociales, aunque también se consideran aquellos procesos no intencionados que puedan generar resultados específicos de inclusión-exclusión. Las dimensiones de este componente son el funcionamiento del sistema democrático y del estado de derecho, por ejemplo: la lucha contra la corrupción, equidad en la administración de justicia, políticas de seguridad humana, las políticas públicas y la operación del mercado.

- El sentido de pertenencia: incluye todas aquellas expresiones psicosociales y culturales que dan cuenta de los grados de vinculación e identificación ciudadana, relativas tanto a la sociedad mayor como a los grupos que la integran, elementos que constituyen el adhesivo básico que permite a la sociedad permanecer junta y que, al mismo tiempo, inciden en las reacciones de los actores frente a las modalidades específicas en que actúan en los diferentes mecanismos de inclusión-exclusión. Las dimensiones de este componente son el multiculturalismo, la no discriminación, el capital social (redes sociales informales, confianza, participación), los valores pro sociales, la solidaridad, las expectativas de futuro, de movilidad social, el sentido de integración y afiliación social.

Así, la cohesión social se produce como un efecto combinado entre las relaciones humanas y la percepción que éstas tienen con respecto a la reducción de las brechas de bienestar. Lo anterior evidencia que para la corriente latinoamericana, la cohesión social se produce como resultado de las acciones gubernamentales, cuyo fin atiende sobre todo, las carencias relacionadas con la inclusión, educación, empleo y la lucha contra la pobreza mediante el fortalecimiento de las capacidades de la sociedad civil como medio para reducir la vulnerabilidad. Por tanto, es necesario identificar los procesos, técnicas y reglas de operación de los programas sociales y de las políticas públicas con el fin de fomentar la cohesión social desde el espacio público mediante el trabajo colectivo, la educación ciudadana y la detección adecuada de las necesidades de una comunidad. (Rivera, 2017b). Así, los programas de gobierno centrados en la educación deberán incproporar como objetivos transversales, la formación de ciudadanía, para comprender la importancia de adoptar normas generalizadas para vivir en sociedad y transformar nuestro entorno para el beneficio colectivo. En particular nos interesa para esta investigación la educación mediada por tecnología, ya que 
Política pública educativa centrada en la tecnología. El caso del programa habilidades digitales para todos en el estado de puebla y su efecto en la formación de cohesión social.

representa un excelente ejemplo de lo anterior, pues produce nuevas normas de comportamiento. En ella, el requisito es el dominio de la tecnología y el uso que hacemos de ésta para difundir el conocimiento, de manera tal que se considera que la política educativa centrada en las tecnologías de información y comunicación tiende a reducir las brechas de conocimiento y en consecuencia produce cohesión social. Por lo cual, abordaremos el estudio de las competencias digitales, las cuales son el conjunto de capacidades, habilidades, destrezas y comportamientos que presenta un individuo en un entorno mediado por la tecnología, donde se le pide hacer frente a situaciones diversas de manera óptima pues se considera que, al tener acceso a la tecnología, los países mejorarán sus condiciones para producir desarrollo. Al respecto, el Banco Mundial publicó en 2005, un reporte titulado Closing the gaps in education and technology donde presenta evidencia que prueba que:

“...cualquier país puede tener una economía exitosa si tiene la capacidad de mejorar sus políticas tecnológicas y educacionales y de adaptarlas a condiciones particulares. Si se aumenta la educación, pero la política no se adapta para promover la accesibilidad a tecnologías nuevas, habrá una disminución en cerebros intelectuales, y por lo tanto no habrá crecimiento." (Gill, et. al, 2005: 8).

Por su parte, la UNESCO ofrece información que establece que “...el mejoramiento en la tecnología y las habilidades resulta en mayor productividad, mejores resultados económicos y en el largo plazo, una mejor calidad de vida para los individuos." (UNESCO, 2009: 8). Por tal razón, el uso de la tecnología en la educación, reducirá las brechas entre las realidades socioeconómicas y la calidad del sistema educativo, por lo que las competencias tecnológicas, les dan facilidad a las personas para desenvolverse socialmente, le permiten sentirse incluido y participar de las decisiones para el bienestar colectivo, generando ventajas competitivas para los paises. La inclusión de las competencias digitales en el currículo docente, tiene varias finalidades entre las que encontramos la integración de los diferentes aprendizajes, es decir, incluye tanto el conocimiento formal como no formal, así mismo, permite al docente integrar sus aprendizajes con diferentes contenidos y utilizarlos en situaciones y contextos necesarios ante alumnos que han crecido en la era de la información 
y que desde pequeños dominan el uso de computadoras y celulares inteligentes. El reto de este tipo de iniciativas gubernamentales es crear un programa académico efectivo que pueda implementarse en todo el país, y que incorpore los componentes necesarios para hacer que personas que no son nativas tecnológicas, puedan adaptarse a un mundo totalmente inmerso en la tecnología.

México presenta condiciones adversas, pues a pesar de ser un país que cuenta con tecnología de punta, el acceso a la misma es costoso y solo una parte de la población tiene acceso a ella. En particular el sector magisterial presenta un reto mayor, pues a la brecha digital se le suma la brecha generacional, en promedio, los profesores que enseñan educación básica son mayores de 50 ańos, por lo que se encuentran en desventaja al momento de utilizar un equipo de cómputo. Aunado a lo anterior, la explicación del mundo dependiente de la tecnología implica sin duda alguna, un cambio en las representaciones sociales que a las generaciones nacidas antes del boom tecnológico se les dificulta comprender. Para este estudio, los datos más representativos respecto a la Política Educativa mexicana centrada en la tecnología son los siguientes:

- Desde 1968 a la fecha, existe en México el programa EDUSAT, que constituye un importante esfuerzo del Gobierno Federal por socializar el uso de las tecnologías de la información y la comunicación en la educación. La creación de este programa buscó mejorar la calidad de la educación y abatir el rezago educativo mediante la propuesta de estrategias que sirvieran de apoyo didáctico a los docentes en servicio, y se dirigió a educación básica, media superior y superior (Gobierno de la República, 2017).

- La Política Pública de Tecnología en México se fortalece en 1972, debido a la necesidad de incrementar la matrícula de estudiantes registrados en el nivel secundaria. El programa de Telesecundaria impulsado por el Presidente Luis Echeverría, fue una respuesta para ampliar la cobertura y llegar a los lugares más remotos transmitiendo la señal educativa vía satélite.

- En 1985, se implementó el Programa de Computación Electrónica en la Educación Básica cuyas siglas son COEEBA. Este programa se orientó a la formación de docentes y alumnos en el uso de la computadora en el aula. Su objetivo final fue la concientización del uso de la computadora como instrumento didáctico. 
Política pública educativa centrada en la tecnología. El caso del programa habilidades digitales para todos en el estado de puebla y su efecto en la formación de cohesión social.

- A partir de 1996, se implementó el programa RED Escolar, el cual consistió en un modelo basado en el uso de la informática educativa y el apoyo de la televisión principalmente a través de la conexión a Internet y EDUSAT. Su propósito fue brindar un modelo tecnológico flexible aplicado a la educación básica para fortalecer el proceso de enseńanza-aprendizaje de maestros y alumnos. Se centró en la formación para el uso de internet, correo electrónico, uso de cds educativos y EDUSAT (SEP, 2010).

- En el año 2006, se implantó el programa Enciclomedia, concebido como un sistema de educación en línea conformado por una base de datos didácticamente diseńada para apoyar la información proporcionada en los libros de texto gratuitos de quinto y sexto de primaria. El programa consistió en dotar de una computadora, un pizarrón electrónico y un proyector a las escuelas públicas del país. En 2008, la Auditoría Superior de la Federación de México, determinó que los resultados del programa no fueron satisfactorios. Las causas se atribuyen sobre todo a la poca capacitación de los maestros en competencias tecnológicas (SEP, 2012).

- En 2011, se instauró el Programa Habilidades Digitales para Todos (PHDT), el cual tras la experiencia de su antecesor Enciclomedia, incorporó cuatro componentes al programa: conectividad, capacitación, infraestructura y acompañamiento (HDT, 2011). Este programa buscó el desarrollo de las tecnologías de la información y comunicación en las escuelas de educación básica, ampliando sus competencias digitales para lograr la inserción de los estudiantes en la sociedad del conocimiento.

- Desde 2012 a la fecha, se inició la colaboración del Gobierno Federal con la Organización para la Cooperación y Desarrollo Económico, denominada por sus siglas: OCDE, con el fin de impulsar a nivel nacional el equipamiento de aulas de medios. Este programa fue implementado por la Asociación Civil UNETE, con el objetivo de empoderar a los docentes del sistema educativo público para que pudieran integrar la tecnología en sus clases, con el apoyo de un formador que acompańaba al docente de manera presencial durante un año escolar.

- En 2013, se dio a conocer un programa piloto para la distribución de Tablets, a alumnos de 5 y 6 de primaria en los Estados de Guanajuato, Morelos y Querétaro, con el fin de continuar con 
el proyecto de integrar a los alumnos en el uso de la tecnología con fines educativos. Este programa a la fecha se encuentra vigente y se ha aplicado en todo el territorio nacional, sin embargo, los resultados han sido son poco favorables, pues la cultura del uso de la tecnología para la educación en México aún es incipiente, tal como lo demuestran los datos de la Encuesta Nacional sobre Disponibilidad y Uso de Tecnologías de la Información en los Hogares publicada por el INEGI en 2016.

- En 2015, como un esfuerzo de integración de la Educación Superior a la formación básica y media, se formaliza la Comunidad Digital de Gestión (CDG) creada como una iniciativa del Gobierno Federal, formada por diversos grupos de expertos universitarios, dedicados al desarrollo de aplicaciones y de recursos educativos, que son concebidos como herramientas de apoyo a los procesos de enseñanza-aprendizaje en la educación superior (CODAES, 2015), esta comunidad brinda los servicios de asesoría, información sobre tendencias, desarrollo de recursos educativos y trayectorias de aprendizaje para la apropiación de una cultura sustentada en el uso de la tecnología para los procesos formativos.

Los esfuerzos por fortalecer la política educativa mediada por la tecnología en México, también se ven afectados por diversas causas:

1.- En México, el uso de la computadora es limitado sobre todo en las zonas rurales del país, donde no existen laboratorios de computación en las escuelas, y el entorno dominante es el campo. La necesidad social no está enfocada a la tecnología. Las condiciones de conectividad del país son limitadas. El acceso a internet es mucho más escaso en las zonas rurales debido a la geografía natural del territorio mexicano. (INEGI, 2016).

2.- Por otra parte, los alumnos en las zonas urbanas, tienen acceso a otros tipos de tecnología además de las computadoras, tal es el caso de tablets y teléfonos inteligentes. También existen plazas públicas que ofrecen servicios de internet gratuito brindado por el gobierno. Sin embargo, las zonas urbanas a pesar de contar con mejores condiciones tecnológicas que las rurales, no tienen desarrollada una cultura en la enseñanza a través de la tecnología, pues el docente promedio, pertenece a una generación que se formó con una educación tradicional, es decir, sin el uso de aparatos tecnológicos. 
Política pública educativa centrada en la tecnología. El caso del programa habilidades digitales para todos en el estado de puebla y su efecto en la formación de cohesión social.

3.- Por ello en el proceso de adopción de una cultura tecnológica ad hoc con la tendencia mundial, se esperaría que los miembros de la comunidad que no son nativos tecnológicos, generen sus propios medios de adaptación, por ejemplo, se podría esperar que la cohesión social, fuera un medio para facilitar la apropiación de conocimientos que permitan la reducción de las brechas relacionadas con el dominio de las competencias digitales.

\section{El Programa Habilidades Digitales para Todos (PHDT) y su implementación en el Estado de Puebla}

Como puede apreciarse en el apartado anterior, el PHDT fue el último en integrar una estrategia educativa integral, con el fin de conformar redes colaborativas para el desarrollo humano de los alumnos a través de la creación y uso de plataformas digitales en cada entidad federativa. Este programa fue creado para dar sentido y razón de ser al uso de herramientas tecnológicas y de comunicación, facilitando la familiarización de los docentes de educación básica con el entorno digital, haciéndolo parte de su vida. Las reglas de operación del programa incorporaron cuatro componentes: El primero fue la creación de un banco de objetos de aprendizaje el cual debía conformarse con el trabajo de los docentes que integraran el programa. El segundo componente fue el acompańamiento pedagógico, para el cual se preveía la participación de un socio educativo en la formación de instructores y la implementación de la certificación en competencias digitales. En casi todas las entidades federativas fueron las universidades estatales quienes fungieron como socios educativos debido a su infraestructura y a su capacidad técnica. El tercer componente fue la inversión realizada en equipamiento y conectividad, la cual buscó dotar a los estados de una red de fibra óptica y del equipo tecnológico necesario para montar aulas de medios útiles. Finalmente, el cuarto componente fue la contraloría social. Este componente integró un elemento novedoso a los anteriores programas de educación en TIC, pues permitió un esquema tipo piramidal para la promoción del uso de las tecnologías en el aula, permitiendo formar una red de colaboración para resolución de dudas, transmisión de conocimientos y asesorías sobre la información brindada en el programa. 
En Puebla, el gobierno estatal firmó un acuerdo de capacitación con la Benemérita Universidad Autónoma de Puebla (BUAP), quien dispuso del personal necesario para la implementación de las acciones enlistadas en las reglas de operación del Programa de Habilidades Digitales para Todos. Para lograr los objetivos de capacitación y certificación en competencias digitales, la BUAP en coordinación con la Secretaría de Educación Pública de Puebla, el Sindicato Nacional de Trabajadores de la Educación sección 23 y 51, la ISTE (International Society for Tecnology Education) y la empresa privada internacional CERTIPORT, encargada de emitir los certificados internacionales, implementaron las estrategias didácticas con el objeto de poder transmitir a los estudiantes poblanos, el manejo de la tecnología dentro del aula. El programa de capacitación HDT, en su versión 2011-2012, capacitó a 15,451 docentes de primaria y secundaria en todas sus modalidades. El programa incorporó la certificación internacional en el estándar IC3 (International Computing Core) a cargo de ISTE y el estándar nacional EC0121 "Elaboración de proyectos de aprendizaje utilizando las tecnologías de la información y comunicación”, emitido por el Consejo Nacional de Certificación en Competencias Laborales (CONOCER), ambos estándares se apoyaron en la plataforma tecnológica de CERTIPORT, la cual fungió como sistema de operación, acopio y control de las evidencias de los participantes para lograr el banco de datos solicitado en el componente número uno.

A través de este programa, el desarrollo de la competencia tecnológica consistió en el tratamiento de la información digital, que permitió disponer de habilidades para buscar, obtener, procesar y comunicar información, y para transformarla en conocimiento, incorporando diferentes habilidades que van desde el acceso a la información hasta su transmisión en distintos soportes. El programa de HDT en Puebla, tuvo los siguientes objetivos:

a) Proporcionar al magisterio poblano elementos tecnológicos sobre el manejo de la información que acompañen el proceso educativo, dentro y fuera de la escuela, para apoyar el aprendizaje de los estudiantes.

b) Contribuir a mejorar el aprendizaje de los estudiantes de educación básica favoreciendo su inserción en la sociedad del 
Política pública educativa centrada en la tecnología. El caso del programa habilidades digitales para todos en el estado de puebla y su efecto en la formación de cohesión social.

conocimiento mediante el desarrollo y uso de tecnologías de la información y la comunicación en el sistema educativo.

Para la implementación del programa en Puebla, se lograron certificar 30 aulas que cumplieron las condiciones necesarias para ser consideradas como aulas evaluadoras mismas que estuvieron distribuidas a lo largo de todo el estado de Puebla y se impartieron 422 cursos de capacitación en las modalidades presencial, mixta y a distancia, en turnos matutino y vespertino, llevando la capacitación por primera vez en la historia a los municipios del interior del estado de Puebla, este hecho fue histórico, sobre todo por tratarse de tecnología y conectividad, pues los programas federales de capacitación a docentes se habían concentrado por tradición en las zonas metropolitanas, haciendo que los profesores del interior del estado tuvieran que trasladarse a la capital, poniendo en riesgo la continuidad en la impartición de su docencia. El programa de capacitación HDT en Puebla, estuvo en funciones del mes de diciembre de 2011 al mes de diciembre de 2012, tiempo durante el cual se llevaron a cabo las siguientes actividades:

- Capacitación y certificación de líderes instructores.

- Capacitación y certificación de líderes evaluadores.

- Capacitación de administradores.

- Certificación de sedes evaluadoras y capacitadoras.

- Diagnóstico de docentes.

- Despliegue del programa de capacitación en Habilidades Digitales para Todos.

- Certificación de docentes en el estándar IC3.

- Certificación de docentes en el estándar EC0121.

El diseño del curso corrió a cargo de ISTE, quien proporcionó los materiales didácticos, la plataforma instruccional y los lineamientos para la capacitación de líderes y docentes del magisterio poblano. Esta actividad se llevó a cabo durante los meses de noviembre de 2011 a enero de 2012, teniendo tres generaciones de líderes instructores. La capacitación de los líderes instructores estuvo a cargo del Coordinador Estatal de ISTE y los docentes fueron evaluados por personal de dicha instancia. Al finalizar el proceso, 94 candidatos se convirtieron en líderes instructores que completaron los requisitos marcados en los lineamientos de ISTE y del programa de 
Habilidades Digitales para Todos. Para lograr la certificación como evaluador, además de cursar el programa de Habilidades Digitales para Todos, los instructores tuvieron que aprobar el programa para ser evaluadores, a través del estándar EC076 del CONOCER, mismo que los acreditó para evaluar competencias laborales. En esta etapa, la capacitación constó de los siguientes elementos:

1.- Participación en el Centro Virtual del CONOCER, donde el candidato cursó cuatro módulos en línea: Derechos de los Usuarios del Sistema Nacional de Competencias; Sistema Nacional de Competencias: propósitos y principios; Dictamen del proceso de Evaluación de Competencias y Evaluación de Competencias y Verificación Interna. El tiempo destinado a este proceso fue de 8 días.

2.- Después de completar la certificación en línea, el candidato se presentó a la capacitación presencial a cargo de personal de la Entidad de Evaluación y Certificación de la BUAP. Esta capacitación tuvo una duración de 21 horas.

3.- Al finalizar la formación presencial, el candidato fue sometido a una evaluación in situ, de manera que al aprobar obtuvo el certificado de competencia como Evaluador.

4.- La entidad de Certificación y Evaluación de la BUAP integró con el trabajo de los evaluadores, los portafolios de evidencia correspondientes a cada uno de los candidatos a líder evaluador, contando con las evidencias físicas del trabajo realizado por los líderes evaluadores.

5.- Finalmente fueron certificados 51 instructores como evaluadores ante el CONOCER. Lo que representó la tercera parte de la meta establecida por la federación.

Con este número de instructores se procedió a capacitar a un total de 15,451 docentes del magisterio poblano, organizados en 416 grupos. Los cursos se impartieron en 32 aulas certificadas con la ayuda de 28 administradores. La numeralia final puede observarse en la Tabla 1. 
Política pública educativa centrada en la tecnología. El caso del programa habilidades digitales para todos en el estado de puebla y su efecto en la formación de cohesión social.

Tabla 1. Estadísticas del Programa de Capacitación en Habilidades Digitales para Todos 2011-2012 en el estado de Puebla

\begin{tabular}{|l|r|}
\hline CONCEPTO & TOTAL \\
\hline Grupos impartidos & 416 \\
\hline Aulas validadas & 32 \\
\hline Docentes diagnosticados & 15,451 \\
\hline Líderes instructores & 51 \\
\hline Líderes evaluadores & 51 \\
\hline Administradores & 28 \\
\hline
\end{tabular}

Fuente: elaboración propia.

Hallazgos relevantes relacionados con la cohesión social en la implementación del Programa Habilidades Digitales para Todos

Las expectativas del programa consistieron en dotar al magisterio poblano de las herramientas tecnológicas que permitieran mejorar la calidad educativa en Puebla mediante el uso didáctico de las tecnologías de la información y la comunicación, permitiendo también el desarrollo y crecimiento de la competitividad en el estado formando estudiantes mejor preparados y con un nivel de razonamiento idóneo para competir en el ámbito local y nacional. Después de impartir 416 repeticiones de la capacitación en el PHDT en todo el Estado de Puebla para 15,451 docentes del magisterio poblano se obtuvieron los resultados en la certificación que se muestran en la Tabla 2.

Tabla 2. Etapa de certificación en el Programa Habilidades Digitales para Todos 2011-2012 en el Estado de Puebla

\begin{tabular}{|l|r|}
\hline CERTIFICACIÓN IC3: CERTIPORT & $\begin{array}{l}\text { TOTAL DE } \\
\text { DOCENTES }\end{array}$ \\
\hline Exámenes de primera oportunidad & 15,451 \\
\hline Exámenes de segunda oportunidad & 7,255 \\
\hline Exámenes equivocados & 93 \\
\hline Personas que excedieron las 7 oportunidades & 57 \\
\hline Exámenes script & 22,771 \\
\hline Personas que presentaron exámenes & 5,241 \\
\hline
\end{tabular}




\begin{tabular}{|c|c|}
\hline Docentes aprobados certificación IC3 & 2,064 \\
\hline CERTIFICACIÓN EC0121: CONOCER & $\begin{array}{l}\text { TOTAL DE } \\
\text { DOCENTES }\end{array}$ \\
\hline Docentes que presentaron exámen & 5,241 \\
\hline Docentes aprobados certificación EC0121 & 753 \\
\hline Docentes convocados al proceso alterno BUAP & 2,500 \\
\hline Diplomas de participación BUAP & 9,556 \\
\hline
\end{tabular}

Fuente: elaboración propia.

Dado que el objetivo de este análisis es demostrar el estado que guarda la cohesión social generada mediante el PHDT, se presenta a continuación los resultados del programa aplicado en el Estado de Puebla, en función de tres indicadores: 1. Distancias o brechas que para el caso del programa HDT corresponden a brechas de conocimiento en particular el uso de la tecnología aplicado a la enseñanza. 2. Mecanismos institucionales, los cuales corresponden a las acciones que, de manera oficial, se generaron durante la implementación del PHDT y 3. Sentido de pertenencia, mecanismo generado por los propios integrantes del programa como estrategia para lograr los objetivos personales y grupales.

En relación a las distancias o brechas, el programa de capacitación en HDT presentó interesantes resultados en cuanto al dominio de las competencias digitales:

- Al integrar el equipo de evaluadores/instructores, se reunió un grupo de 170 docentes de nivel básico propuestos por la Secretaría de Educación Pública de Puebla, y 20 docentes de educación superior propuestos por la BUAP. Las diferencias encontradas entre ambos grupos fueron las siguientes: El grupo de universitarios fue mejor evaluado en técnicas propias del dominio de la materia, mientras que los docentes del nivel básico obtuvieron mejores resultados en el dominio de la didáctica. Esto significó que, durante el proceso de evaluación, los docentes universitarios fueran mejor evaluados en los exámenes, mientras que los docentes de educación básica obtuvieron mejores resultados en la presentación del proyecto (objeto de aprendizaje). 
Política pública educativa centrada en la tecnología. El caso del programa habilidades digitales para todos en el estado de puebla y su efecto en la formación de cohesión social.

- Respecto al dominio previo de la tecnología, el 49 por ciento de los docentes que participaron en los cursos para instructores provenían de municipios del interior del estado. La población estuvo conformada por $63 \%$ mujeres y $37 \%$ hombres.

- Del total de mujeres participantes como líderes instructoras, el $72 \%$ participó en la modalidad presencial, el $21 \%$ en mixta y solo el $7 \%$ en modalidad de autoestudio, mientras que del total de hombres que participaron en esta etapa del proyecto HDT, el 59\% participó en modalidad presencial, el $27 \%$ en mixta y el $14 \%$ en modalidad de autoestudio.

Los resultados durante la capacitación de los 15,451 docentes que participaron en el programa arrojaron lo siguiente:

- Del total de 15,451 docentes que iniciaron el proceso solo el $62 \%$ terminó el programa de capacitación, de los cuales solo el $21.59 \%$ logró la certificación en IC3 mientras que sólo el $8 \%$ obtuvo la certificación en el estándar ECO121.

- El 80\% de los participantes no estaba familiarizado con el uso de la computadora e internet.

- El 90\% de los participantes no contaban con un correo electrónico habilitado.

- El 80\% de los participantes no contaba con una computadora en casa y en los casos donde si tenían acceso a equipo de cómputo, su uso era compartido, sobre todo con sus hijos.

- La comunicación vía electrónica no representó un medio de comunicación para el sector magisterial, pues el uso de la tecnología en el aula no fue una prioridad en la planeación educativa tradicional.

- El área donde los docentes obtuvieron resultados más bajos al finalizar la capacitación fue en la elaboración de su proyecto de aprendizaje utilizando las tecnologías de la información y comunicación, debido a que la elaboración del proyecto requería dedicación de tiempo completo y un manejo intermedio en el uso de las TICS. Para casi la totalidad de los participantes, la capacitación fue el primer contacto con el uso de internet y computadoras para fines educativos.

- Los docentes percibieron como muy difícil la elaboración del proyecto en línea (objeto de aprendizaje). Pues requería además de las herramientas técnicas y de comunicación aprendidas durante el programa de capacitación en HDT, los conocimientos necesarios para desarrollar el contenido. 
En cuanto a los mecanismos institucionales, corresponden a las acciones ejecutadas por los distintos actores institucionales, para este programa, el equipo operativo integrado por SEP, BUAP, CERTIPORT e ISTE. Los principales mecanismos de inclusión y sus efectos se describen a continuación:

- La convocatoria para invitar a los docentes del magisterio poblano a realizar el diagnóstico inicia, se realizó en toda la entidad poblana, con la ayuda del personal de SEP en los diferentes niveles educativos; Esta convocatoria tuvo poca respuesta pues los docentes se encontraban escépticos con respecto al uso de las tecnologías, pues su participación en el programa no estuvo condicionada (es decir, no fue obligatoria).

- Después de insistir en varias ocasiones con invitaciones y llamados por parte de los diversos niveles académicos, el ejercicio dio como resultado que los principales promotores del programa fueran precisamente los docentes que fungieron como líderes instructores. Es importante mencionar para fines del análisis de la cohesión social, que el docente del magisterio poblano tiene una tradición de comunicación oral muy arraigada, lo cual fue la mejor estrategia para involucrar a otros docentes que inicialmente no estuvieron dispuestos a cursar el programa.

- El tiempo previsto para el desarrollo de este proyecto fue de 10 días hábiles, desde el inicio de la capacitación hasta la conclusión del programa, tiempo que no fue suficiente para procesar la información brindada en la capacitación y aplicarla en un caso práctico.

- Se reconocen dos causas que provocaron la no certificación; la primera fué que la participación en el programa no tuvo un carácter obligatorio; la segunda consistió en que el docente no entregó el proyecto solicitado. (Del total de docentes no aprobados, el 60\% no cumplió con la entrega de este requisito, dándose por vencidos, debido al tiempo establecido para la entrega del mismo).

Referente al sentido de pertenencia, incluye las expresiones psicosociales y culturales que dan cuenta de los grados de vinculación e identificación ciudadana, para el caso del magisterio poblano, la cohesión social se refleja en las acciones realizadas como estrategias emergentes de un grupo de docentes para el logro de sus objetivos individuales. El sentido de pertenencia en el PHDT en Puebla, pueden identificarse con mucha claridad: 
Política pública educativa centrada en la tecnología. El caso del programa habilidades digitales para todos en el estado de puebla y su efecto en la formación de cohesión social.

- De los 9556 docentes que aprobaron la certificación en IC3, el $75 \%$ asistió a reuniones de autoestudio organizadas por ellos mismos, estas actividades consistieron en la formación de equipos donde uno de los participantes más habilitados enseńó a sus colegas aquellas actividades más complicadas. Debido a lo anterior, la BUAP tuvo que habilitar áreas de estudio activas las 24 horas del día, pues los espacios programados estaban destinados a la capacitación formal. Este es un ejemplo del sentido de pertenencia que produce una reacción institucional.

- El $100 \%$ de los asistentes a las actividades de autoestudio aprobó en una segunda vuelta el examen de certificación en IC3, comprobando con ello, la existencia de los beneficios de la cohesión social en el desarrollo de competencias digitales.

\section{Reflexiones finales}

Tras el análisis mostrado, la cohesión social resulta de la necesidad de un grupo particular para obtener un objetivo común. En 2011 se puso en marcha el Programa de Capacitación en Habilidades Digitales para Todos, el cual incorporó componentes que no se habían integrado antes como la contraloría social, la cual tuvo un efecto detonante en el interés de los docentes en participar en dicha capacitación en el Estado de Puebla. Además de lo anterior, la auto gestión de los docentes del magisterio poblano fue el factor clave para que el total de docentes que participaron en los grupos de autoestudio obtuvieran la certificación IC3. Estas dos actividades (la contraloría social y los grupos de autoestudio) no se encontraban planeadas dentro del esquema de capacitación siendo un hallazgo relevante que prueba la existencia de cohesión social en la política educativa centrada en el uso de la tecnología, la cual permite la reducción de brechas de conocimiento, la implementación de mecanismos institucionales y el desarrollo de sentido de pertenencia, mismo que se genera de manera natural en los grupos de individuos producto de la socialización.

Los hallazgos de esta investigación, resaltan la existencia de una reducción limitada en las brechas de conocimiento en particular en el uso de la tecnología a través de la aplicación del PHDT, pues solo el $21.59 \%$ de los docentes que participaron en la capacitación ob- 
tuvieron la certificación internacional. Sin embargo, es predecible que la propia estructura del programa establecida en las reglas de operación sea una de las principales causas, debido sobre todo a que la capacitación y certificación tuvo una duración total de 21 días establecidos de acuerdo a las normas internacionales, donde se espera que los profesores tengan conocimientos previos del uso de la computadora. Para el caso estudiado, los docentes poblanos no contaban con preparación previa en el uso de la computadora ni contaban con acceso a email o internet, por tanto, percibieron como muy difícil la elaboración del proyecto en línea (objeto de aprendizaje).

En cuanto a los mecanismos institucionales, los hallazgos comprueban que su desarrollo en el Estado de Puebla tuvo efectos interesantes, pues se encontraron estrategias alternas para involucrar a los docentes en la capacitación a pesar de que el programa no tuvo carácter obligatorio: La participación integrada de los líderes instructores, fue un factor clave para que se alcanzaran las metas de capacitación; La disposición de la SEP y de la BUAP como socio educativo para proveer a los docentes de espacios para el autoestudio que contaran con el mismo acondicionamiento tecnológico que las aulas certificadas, fue un esfuerzo que se tradujo en el éxito del programa. Es importante mencionar que Puebla fue uno de los pocos estados del país que logró las metas de capacitación. El hallazgo más relevante del PHDT en cuanto a cohesión social fue el desarrollo de estrategias de autogestión producto del sentido de pertenencia entre los miembros del magisterio poblano que participaron en la capacitación, pues los grupos de autoestudio no estaban considerados dentro de la planeación oficial. El 75\% de los profesores certificados, asistió a esta actividad.

En este sentido, se observa que, para el escenario poblano, la inclusión tecnológica es condición para el crecimiento y el desarrollo humano y social, pues, aunque las expectativas de los grupos sociales son limitadas, se generan subgrupos con un plan específico, en este caso, fue notoria la asociación de docentes que deseaban obtener la certificación internacional, no fue así para el caso de la certificación nacional, como puede apreciarse en apartados anteriores. Finalmente, se concluye que la actualización en temas tecnológicos debe integrarse en la construcción de un currículo orientado a las competencias tecnológicas que contribuya a la 
Política pública educativa centrada en la tecnología. El caso del programa habilidades digitales para todos en el estado de puebla y su efecto en la formación de cohesión social.

realización y desarrollo personal de los alumnos, pues estos serán capaces de apropiarse de aprendizajes permanentes, así mismo, se desarrollará una ciudadanía activa que impacte en el mejoramiento de la integración social. Si bien es cierto que este esfuerzo nacional obligó a los directivos estatales a realizar acciones de coordinación interna, también fue un hecho que la participación de actores sociales, como las Universidades Públicas, facilitaron la implementación de las acciones gubernamentales, al ser considerados integrantes activos de la sociedad.

\section{Referencias bibliográficas}

Botterman, S., Hooghe, M. y Reeskens, T. (2012). 'One Size Fits All'? An Empirical Study into the Multidimensionality of Social Cohesion Indicators in Belgian Local Communities. Urban Studies, 2012, vol. 49, no 1, p. 185-202.

Carron, A. V. y Spink K. S. (1995). The group size-cohesion relationship in minimal groups. Small Group Research. Vol. 26, no. 1. 1995. P. 86-105.

Consejo Nacional de Evaluación de la Política de Desarrollo Social. CONEVAL. (2012). Informe de Pobreza en México, el País los Estados y sus Municipios. México. CONEVAL.

Comisión Económica para América Latina y el Caribe. CEPAL. (2017). Informe Anual sobre el Progreso y los Desafíos regionales de la Agenda 2030 para el Desarrollo Sostenible en América Latina y el Caribe. CEPAL. 27 de abril de 2017. Consultado el 17 de septiembre de 2017. Recuperado de: http://repositorio.cepal.org/bitstream/handle/11362/41173/7/S1700475_es.pdf

Comisión Económica para América Latina y el Caribe. CEPAL. (2007). Un sistema de indicadores para el seguimiento de la cohesión social en América Latina. Santiago de Chile, Naciones Unidas. CEPAL.

Comunidades Digitales para el Aprendizaje en la Educación Superior. CODAES. (2015). Lo más reciente. Consultado el 5 de agosto de 2017. Recuperado de 2017 de: https://www.codaes.mx

Cramm, J. M.; Van Dijk, H. M.; Nieboer, A. P. (2013). The importance of neighborhood social cohesion and social capital for the well being of older adults in the community. The Gerontologist. Vol. 53, no. 1, 1 February 2013, p. 142-152. 
Demireva, N., Mcneil, R. (2012). Immigration, Diversity and Social Cohesion. The Migration Observatory at the University of Oxford. University of Oxford. UK. Briefing. COMPAS.

Gill, I., Guasch J., Maloney W., Perry G., Chady N. (2005). Cerrando la brecha en educación y tecnología. Serie Desarrollo para Todos \# 9. Colombia. Banco Mundial.

Gobierno de la República. (2017). RED Edusat. Consultado el 1 de septiembre de 2017. Recuperado de: http://www.televisioneducativa. gob.mx/red-edusat

Instituto Nacional de Estadística y Geografía. INEGI. (2016). Usuarios de Tecnologías de Información y Usuarios de Internet según principales usos. Base de Datos de la Encuesta Nacional sobre Disponibilidad y Uso de Tecnologías de la Información en los Hogares (ENDUTIH). México. INEGI.

Jenson, J. (2010). Defining and measuring social cohesion. London. UK. United Nations. Research Institute for Social Development. Commonwealth Secretariat.

Juul, S. (2010). Solidarity and Social Cohesion in Late Modernity: A question of Recognition, Justice and Judgement in Situation. European Journal of Social Theory, vol. 13, 2010, no. 2 p. 253-269.

KawachI I. y Kennedy B. P. (1997). Health and social cohesion: why care about income inequality? BMJ. 1997 Apr 5; vol. 314, no. 7086. p. 1037-1040.

Programa Habilidades Digitales para Todos. PHDT. (2011). Reglas de operación del Programa Habilidades Digitales para Todos. Consultado el 12 de agosto de 2017. Recuperado de http://www.hdt.gob. $\mathrm{mx} /$ Paginas/home.aspx

Rivera, C. (2017a). Cohesión Social en las Agendas Mundiales. Conferencia Dictada el 14 de mayo de 2017. Puebla, México. Instituto de Ciencias de Gobierno y Desarrollo Estratégico. BUAP.

Rivera, C. (2017b). Reflexiones para el estudio de la Cohesión Social en torno al desarrollo de México y América Latina. En: Rivera C. (coord.). (2017). Cohesión Social para el Desarrollo de México y América Latina. México, BUAP, CONACYT, SEDESOL.

Secretaría de Educación Publica. SEP. (2012). Libro Blanco Programa Enciclomedia 2006-2012. Consultado el 1 de septiembre de 2017. Recuperado de : http://www.sep.gob.mx/work/models/sep1/Resource/2959/4/images/LB\%20Enciclomedia.pdf 
Secretaría de Educación Publica. SEP. (2010). Programa Red Escolar.

Consultado el 31 de agosto de 2017. Recuperado de: http://www.seebc.gob.mx/redescolar/

Unión Europea. UE. (2013). Programa para la Cohesión Social en América Latina. UE. Consultado el 17 de octubre de 2013. Recuperado de http://www.eurosocial-ii.eu/eurosocial/que-hacemos/cohesionsocial

Organización de las Naciones Unidad para la Educación, la Ciencia y la Cultura. UNESCO. (2009). Medición de las tecnologías de la información y la comunicación (TIC) en educación -manual del usuario. Montreal, Canadá. UNESCO-IEU. Consultado el 2 de agosto de 2017. Recuperado de: http://unesdoc.unesco.org/images/0018/ 001883/188309s.pdf

Vasta, E. (2010). The controllability of difference: Social cohesion and the new politics of solidarity. Ethnicities, vol. 10, no 4, 2010. p. 503521.

Warburton, K. y Lazarus, J. (1991). Tendency distance models of social cohesion in animal groups. Journal of Theoretical Biology, (21 June 1991), vol. 150, no 4, p. 421-563.

Wey, T. W., y Lumstein D. T. (2010). Social Cohesion in yellow Bellied Marmots is established through age and kin structuring. Animal Behaviour, 2010, vol. 79, no 6, p. 1343-1352.

Wilkinson, R. G. (1999). Inequialities and Health. Income Inequality, Social Cohesion, and Health: Clarifying the Theory. A Replay to Muntaner and Lynch. International Journal of Health Services. Vol. 29. No. 3. 1999. p. 525-544. 